\title{
Design for Real-Time Monitoring System of High Oxygen Modified Atmosphere Box of Vegetable and Fruit for Preservation
}

\author{
Zhanli Liu ${ }^{1}$, Congcong Yan ${ }^{1}$, Xiangyou Wang ${ }^{1, *}$ and Xiangbo Han $^{2}$ \\ ${ }^{1}$ School of Agriculture and Food Engineering, \\ Shandong University of Technology, P.R. China \\ ${ }^{2}$ College of Computer Science and Technology, \\ Shandong University of Technology, P.R. China \\ wxy@sdut.edu.cn
}

\begin{abstract}
According to the disadvantages of traditional box for preservation, including low oxygen and high carbon, a new control system of high oxygen is designed. This paper presents the design and implementation method in this system. The system combines traditional PID control with fuzzy control which can adjust parameters of the whole system automatically. The box can control the dynamic content of oxygen and carbon dioxide, and monitor nitrogen flow, temperature and humidity real-timely so that preservation time can be prolonged. It also can collect and keep the data of the dynamic content of oxygen and carbon dioxide which suits for fruit and vegetable for preservation. The former environment can be reappeared when need. The system works steadily and has strong functions.
\end{abstract}

Keywords: High oxygen; Modified atmosphere box; Real time monitor; Design.

\section{Introduction}

Day, the British scholar for the first time made clearly the application of high-oxygen $\left(>70 \% \mathrm{O}_{2}\right)$ in modified atmosphere packaging of fresh-cut vegetables and fruits in 1996. Domestic and foreign research of high-oxygen $\left(21 \%-100 \% \mathrm{O}_{2}\right)$ on the effect of postharvest gradually increased, and the treatments of high oxygen are expected to play an important role in fruit and vegetable storage[1]. Research has shown that high-oxygen treatment of fruits and vegetables can reduce the respiration and ethylene production, slow their browning, and improve the preservation effects[2-4]. The application of high-oxygen or even pure oxygen modified atmosphere technology on fruit and vegetable preservation has aroused close attention. So it is necessary to make a study of a reliable and stable high oxygen modified atmosphere control system for further development of high-oxygen fresh-keeping equipment. However, the impact mechanism of the high oxygen on the postharvest physiology and quality is not indepth, so it severely limits the development of high oxygen storage[5]. Thus, most of

\footnotetext{
* Corresponding author.
} 
high oxygen experiments lack of accurate monitoring of oxygen, carbon dioxide, nitrogen flow rate, temperature and humidity, and can not control stably the environment of high oxygen. In this paper, the self-made high oxygen experimental apparatus were studied, and a data acquisition system and related test equipment were developed providing a scientific basis for high oxygen modified atmosphere theory.

\section{Experimental Device of High Oxygen Modified Atmosphere Box}

Central processing section formed by a microprocessor is the heart of the whole control system, which completes the functions of data acquisition and processing, keyboard and display processing and system control. Temperature and humidity transmitter is mainly to measure gas concentration of the tested environment, and output the standard current signal corresponding to the surrounding gas concentration after the signal processed by the internal data processing. Gas flow meter part is mainly to measure the volume of the input nitrogen. It can select 4-20mA output, level output, or pulse output module according to needs. Analog multiplexer switch is mainly to turn the switch channel between separate ways analog and $\mathrm{A} / \mathrm{D}$ converter, allowing one way analog signal input to the A/D converter in a specific period of time, and it can achieve the purpose of time-conversion to reduce the number of $\mathrm{A} / \mathrm{D}$ converter and reduce costs. Current transfer voltage circuit is used to change the transmitter and flow meter standard output current signal into the corresponding voltage signal and conduct the follow-up signal processing. The role of sample retainer is used to maintain analog signal voltage of the $\mathrm{A} / \mathrm{D}$ converter input constant during the conversion period, then ensure a higher conversion precision, and greatly increase the collection frequency of data collection. Some data memory stores the measured data immediately and preserves the measured environmental data for long-term. And data can be transferred out to external memory by a data interface, facilitating accurate analysis. Optical isolation part completes the isolation and amplification from weak signal to strong signal. The conversion of manual control and auto-control can be set by Panel.

In the case of automatic failure or manual intervention (for example, using the manual control can quickly reach steady state before reaching the set high oxygen concentration), some manual control part can control all of the conditioning systems of the atmosphere storage environment. Program memory is used to store system procedures. Solenoid valve is used to control nitrogen access.

\section{Working Principle of High Oxygen Modified Atmosphere Box}

The concentration of oxygen and carbon dioxide, temperature and humidity of box are measured by the corresponding transmitter. Transmitter output signal is transmitted to the microprocessor after a series of treatments, and compared with the set initial values, when any kind of gas concentration is below the set value, appropriate instructions will be issued by the microprocessor, then the electric value corresponding is opened with the gas to let that kind gas in. By controlling the opening of the electric valve to control the air flow rate, when the concentration closes to the set value, the microprocessor controls the corresponding procedure to reduce the opening to prevent the excessive ventilation and avoid its concentration exceeding the set value. When the value of 
any gas concentration is higher than the set alarm value, the system alarms, and the corresponding unit of the micro-processor will give appropriate instructions, so that the corresponding electric valve of the gas will be closed, and the nitrogen valve will be opened, by inletting into constant flow of nitrogen the concentration of this gas will be reduced. When the concentration of this gas closes to the set value, the microprocessing time relay control valve will close for 10 seconds, leaving some buffer time to avoid excessive nitrogen filled. Then process it according to the measured actual situation. The gases coming out from nitrogen generator, oxygen, and carbon dioxide bottles pass into the inlet pressure regulator box, and then pass into the test box. The gas cylinder export pressure of the nitrogen generator, oxygen and carbon dioxide bottles can be set much higher, and then adjust the inlet air pressure to the required. This can avoid failing to reach the export settings when the cylinder pressure is insufficient, and the pressure can be precisely adjusted by the intake air pressure tank.

\section{Data Acquisition System}

Oxygen sensor is most important one of all the sensors of the system, and it has a very big impact on the system, so its selection is of great importance. It must meet the fast, reliable and accurate measurement of the oxygen concentration. Now commonly used oxygen sensor is electrochemical sensors. According to the principle, $\mathrm{CO}_{2}$ sensor can be divided into electrochemical $\mathrm{CO}_{2}$ sensor and infrared $\mathrm{CO}_{2}$ sensor, the former is cheaper, but the accuracy is lower, and life is shorter, and preheat time is longer than the latter. Compared to carbon dioxide transmitter, carbon dioxide sensor is low precision, poor linearity, less function, and also needs to set up a dedicated signal processing devices for filtering and V/I conversion for processing, so the costs are increased. In view of the advantages of the transmitter, we use the transmitter (with imported high-grade infrared sensor) to replace the specialized sensors.

\section{Experimental Debugging}

Agaricus bisporus were used as the materials to test the stability of the high oxygen experimental box. The concentrations of oxygen and carbon dioxide were set

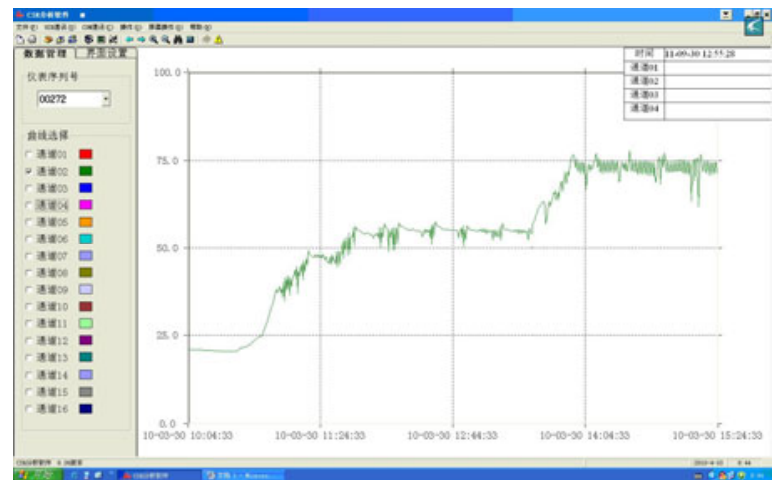

Fig. 1. Change trends of oxygen in box 


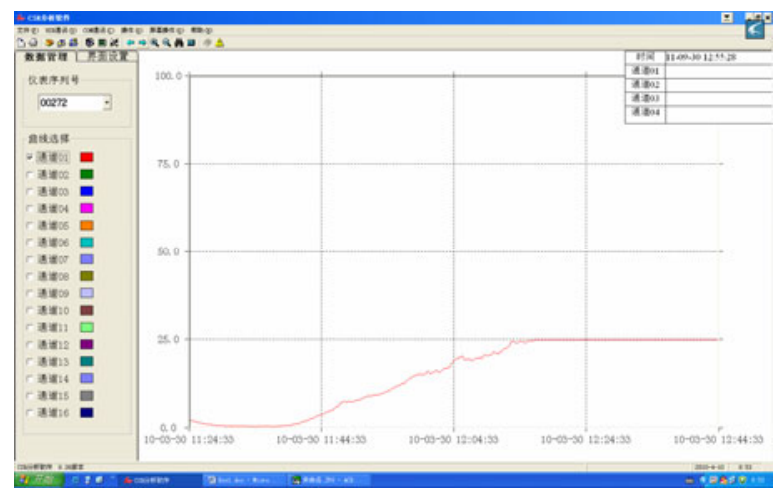

Fig. 2. Change trends of carbon dioxide in box

respectively to $75 \%$ and $25 \%$, and stabilized at a predetermined value (fig. 1 and fig. 2). From the results, the high oxygen modified box can real-time control the dynamic contents of oxygen and carbon dioxide.

Acknowledments. This study was supported by the National Natural Science Foundation of China (No. 30871757).

\section{References}

[1] Zheng, Y.H.: Superatmospheric oxygen and postharvest physiology of fresh fruits and vegetables. Plant Physiol. Comm. 38(1), 92-97 (2002)

[2] Li, P.X., Wang, G.X., Liang, L.S., et al.: Effects of high oxygen treatments on respiration intensity and quality of 'DongZao' jujube during shelf-life. Transactions of the Chinese Society of Agricultural Engineering 22(7), 180-183 (2006)

[3] Escalona, V.H., Verlinden, B.E., Geysen, S., et al.: Changes in respiration of fresh-cut butterhead lettuce under controlled atmospheres using low and superatmospheric oxygen conditions with different carbon dioxide levels. Postharvest Biology and Technology 39, 48-55 (2006)

[4] Conesa, A., Verlinden, B.E., Artés-Hernández, F., et al.: Respiration rates of fresh-cut bell pepper under superatmospheric and low oxygen with or without high carbon dioxide. Postharvest Biology and Technology 45, 81-88 (2007)

[5] Liu, Z.L., Wang, X.Y., Zhu, J.Y., et al.: Progress in effects of high oxygen on postharvest physiology and quality of fresh fruits and vegetables. Transactions of the Chinese Society for Agricultural Machinery 40(7), 112-118 (2009) 